\title{
Multicenter Validation of Three-Dimensional Echocardiographic Quantification of the Left Heart Chambers using Automated Adaptive Analytics
}

\author{
Diego Medvedofsky ${ }^{1}$, Roberto M.Lang ${ }^{1}$, Mihaila Amzulescu², Covadonga Fernández-Golfín ${ }^{3}$, Rocio \\ Hinojar ${ }^{3}$, Mark J. Monaghan ${ }^{4}$, Joseph Reiken ${ }^{4}$, Masaaki Takeuchi ${ }^{5}$, Wendy Tsang ${ }^{6}$, Jean-Louis \\ Vanoverschelde $^{2}$, Mathivathana Indrajith ${ }^{4}$, Lynn Weinert $^{1}$, Jose Luis Zamorano ${ }^{3}$, Victor Mor-Avi ${ }^{1}$ \\ ${ }^{1}$ University of Chicago, Chicago, Illinois, US \\ ${ }^{2}$ Saint-Luc University Hospital, Brussels, Belgium \\ ${ }^{3}$ University Hospital Ramón y Cajal, Madrid, Spain \\ ${ }^{4}$ King's College Hospital, London, UK \\ ${ }^{5}$ University of Occupational and Environmental Health, Kitakyushu, Japan, \\ ${ }^{6}$ Toronto General Hospital, University of Toronto, Toronto, Ontario, Canada
}

\begin{abstract}
Although recommended by the current guidelines, $3 D$ echocardiographic (3DE) quantification of the cardiac chambers in clinical practice has been lagging, because of time-consuming analysis. We recently validated an automated algorithm that measures left atrial (LA) and left ventricular $(L V)$ volumes and ejection fraction $(E F)$. This study aimed to determine its accuracy and reproducibility in a multicenter setting. 180 patients underwent 3DE imaging (Philips) at 6 sites. Images were analyzed using automated HeartModel (HM) software with endocardial border correction when necessary, and also by conventional manual tracing. Measurements were performed independently by each site and by the Core Laboratory (CL), whose measurements were used as a reference. Inter-technique comparisons included HM measurements by the sites with and w/o corrections against manual tracing by CL. Intra-technique comparisons included HM measurements by the sites against those by CL (with and w/o corrections, respectively). Inter-technique comparison w/o corrections showed strong correlations, with the automated technique slightly underestimating $L V$ volumes. Corrections were either unnecessary or minimal in most patients, and affected the measurements minimally. Intra-technique comparisons with corrections showed better correlations and smaller biases than the inter-technique comparison. All automated measurements with corrections were more reproducible than manual measurements. Automated volumetric analysis of left-heart chambers is an accurate alternative to conventional methodology, which yields almost the same values across laboratories and is more reproducible. This technique may contribute towards full integration of $3 D E$ quantification into clinical routine.
\end{abstract}

\section{Introduction}

Over the past decade, real-time 3D imaging has become an integral part of the echocardiography landscape because of its proven advantages over 2D imaging in multiple areas. With the availability of 3DE equipment and analysis software and the rapidly growing body of knowledge, this novel methodology is earning its place as the new standard in many areas. One area where its advantages over 2D echocardiography (2DE) are well recognized is the quantification of cardiac chambers' size and function with increased accuracy and reproducibility [1-2]. Although recent guidelines recommend the use of 3DE quantification of left-heart chambers [3], the current software implementation of this approach relies on extensive user input, making it too time consuming for a busy clinical laboratory. As a result, this methodology has not been fully integrated into the routine clinical work [1, 4]. To bridge this gap, we recently tested the feasibility of a new automated approach for left-heart chamber quantification based on an adaptive analytics algorithm. We showed good accuracy and reproducibility, and improved speed of analysis, compared to the conventional 3DE methodology [5]. As the development of the algorithm continued, multiple refinements were made, resulting in improved endocardial boundary detection in a larger percentage of patients. We hypothesized that in its current form, the automated $3 \mathrm{DE}$ analysis that simultaneously quantifies LV and LA volumes and LV EF would universally provide accurate and reproducible measurements, and thus would be suitable for widespread clinical use. To test this hypothesis, we designed a multicenter validation study, which included comparisons of the automated measurements made by the participating sites with and without corrections against two sets of 
reference values, generated by trained personnel at the CL: conventional 3DE methodology (inter-technique comparisons), and the new automated measurements (intra-technique comparisons).

\section{Methods}

We studied patients referred for clinically indicated echocardiograms for a wide range of conditions, who underwent transthoracic 2DE and 3DE imaging at 6 institutions. Participating sites underwent brief training by the developers of the new software. Each institution enrolled 30 patients into 4 groups defined according to the biplane 2DE LV EF (1: $\leq 20 \%$, 2: 21-40\%, 3: 41-55\%, 4: $>55 \%$ ), to ensure a wide range of chamber size and function. 3DE images of these 180 patients (age $57 \pm 18$ years) were analyzed by both the enrolling site and the $\mathrm{CL}$ to measure $\mathrm{LV}$ volumes and $\mathrm{EF}$ and LA volume (LAV) using two 3DE techniques: the conventional manual analysis and the new automated analysis.

\subsection{Echocardiographic imaging and analysis}

Imaging was performed using the EPIQ system (Philips) with a matrix array transducer (X5-1), and included: 2DE images in the apical 2- and 4-chamber views, and a wide-angled "full-volume" 3DE datasets (HM ACQ key on EPIQ 7C). Care was taken to include the entire LV and LA cavity within the images. Images were optimized to obtain the highest possible frame rate.

All 3DE images were analyzed by both the site and the CL. Measurements were performed by independent experienced investigators and included: LV end-diastolic (ED and end-systolic (ES) volumes (EDV, ESV), EF and LAV by two 3DE techniques: the new automated analysis (Philips, HeartModel (HM)) and the conventional manual analysis (Philips, 3DQ). The readers were blinded to the results of all previous analyses.

The automated 3DE software was described in our recent publication [5]. Briefly, the software simultaneously detects LV and LA endocardial surfaces using an adaptive analytics algorithm, which uses knowledge-based identification to orient and locate chambers and patient specific adaptation of endocardial borders. The algorithm automatically creates ED and ES 3D casts of the LV cavity and an ES cast of the LA cavity, from which LV and LA volumes are derived directly without geometric assumptions. Because of the fully automated nature of the algorithm, it has a deterministic convergence response, thus yielding the exact same result when repeating the analysis on the same dataset. However, manual corrections of the resultant $\mathrm{LV}$ and LA endocardial surfaces are possible, when the operator judges the automatically detected surface as inadequate. This is achieved by displaying the LA and LV contours on 4-, 3and 2-chamber cut-planes extracted from the $3 \mathrm{DE}$ datasets and allowing the user to edit the contours to optimize their position (Figure 1). The LV casts can be edited by either changing the entire border globally (dilating or contracting the entire surface uniformly by the same distance) or by editing it regionally. In contrast, the LA cast can be edited only regionally.

Manual 3DE measurements of LV EDV and ESV volumes and LAV were performed using commercial software (3DQ, QLAB, Philips). The following steps were performed on the ED and ES frames chosen by the automated technique. First, the user selected from the 3DE dataset non-foreshortened apical 2- and 4-chamber views focused on the relevant chamber ( $\mathrm{LV}$ or $\mathrm{LA}$ ), in which the long-axis dimension of the respective chamber was maximized. Then, 4 mitral annular points were marked in each of the views, and an additional point was placed to mark either the apex for LV analysis, or the most distal point on the atrial roof for the LA analysis.

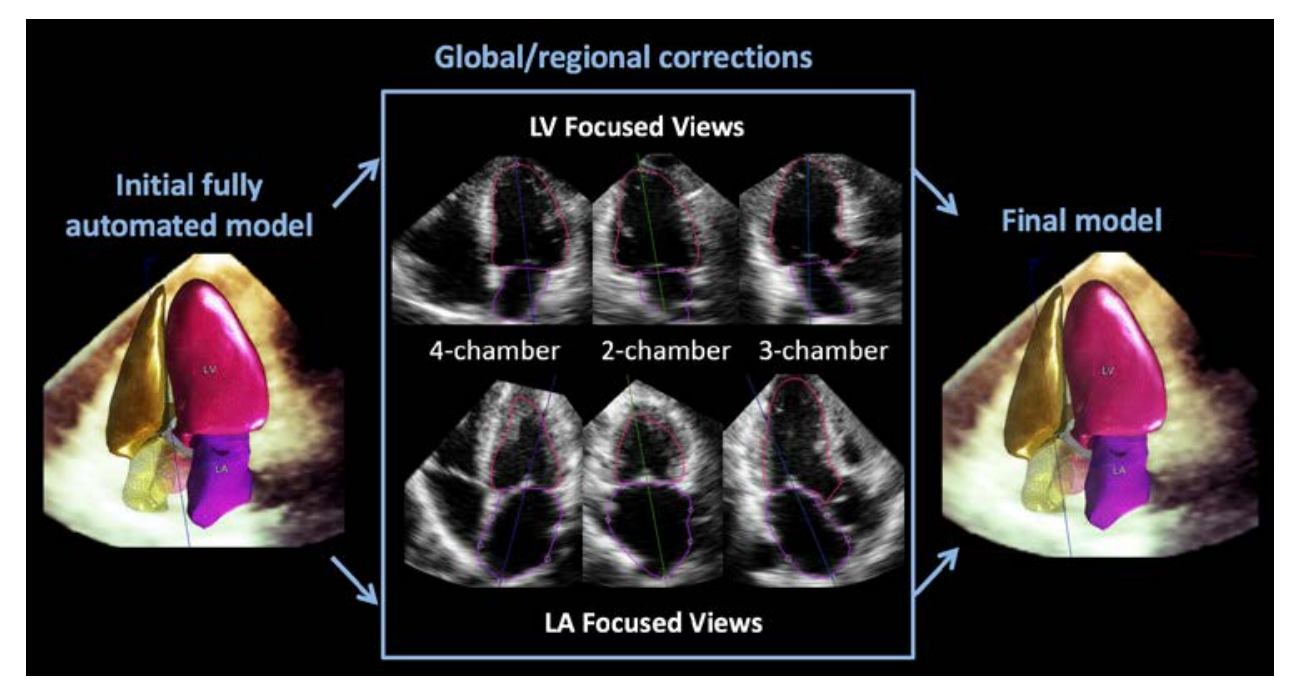

Figure 1. Automated technique for left-heart 3D chamber quantification. Following initial fully-automated detection of LV and LA endocardial surfaces (left), the software allows the user to perform manual corrections of the endocardial boundaries when needed (center), resulting in final 3D casts of the cardiac chambers. The optional correction are performed in non-foreshortened 2D planes showing focused long-axis views of both chambers, both automatically extracted from the 3D dataset. 
The endocardial border was automatically generated, edited as needed and used to calculate LV EDV, ESV and LAV.

The reproducibility of the volume measurements using both 3DE techniques (manual and automated) was tested in a randomly selected subgroup of 90 patients. The same 3DE datasets were reanalyzed using both programs by the same investigator blinded to all prior measurements. These repeated measurements were used to determine the intra-observer variability. Inter-observer variability was assessed by comparing measurements performed by the enrolling sites and the CL. Variability was expressed as an absolute difference between the corresponding pair of repeated measurements in percent of their mean.

\subsection{Statistical analysis}

For each parameter, the intra- and inter-technique comparisons included linear regression and Bland-Altman analyses. The calculated biases were also expressed in percent of the mean measured value of each parameter. Paired t-tests were used to verify the significance of the biases. Values of $\mathrm{p}<0.05$ were considered significant.

\section{Results}

Of the patients submitted by the sites to the CL, $10 \%$ were rejected because of inadequate image quality, and their slots were repopulated with new patients. Intertechnique comparisons showed excellent agreement between the automated 3DE volume measurements performed by the sites without boundary corrections and the CL manual 3DE measurements, with measured values being similar between the two techniques (Table 1, rows 1- 2). The correlations for the volumes were excellent ( $\mathrm{r}=0.97,0.97$, and 0.96 for LV EDV, ESV and LAV, respectively), while that for EF was lower ( $\mathrm{r}=0.88)$. Volumes were underestimated with small biases $(-14$ $\pm 20 \mathrm{ml}$ for LV EDV, $-6 \pm 20 \mathrm{ml}$ for ESV and $-9 \pm 10 \mathrm{ml}$ for LAV), and the bias in EF was also minimal (-2 $\pm 7 \%)$.

\begin{tabular}{|ccccc|}
\hline & EDV $(\mathrm{ml})$ & ESV $(\mathrm{ml})$ & EF $(\%)$ & LAV $(\mathrm{ml})$ \\
\hline Core Lab's Manual values & $203 \pm 83$ & $132 \pm 80$ & $39 \pm 15$ & $89 \pm 36$ \\
\hline Bites' HeartModel values without corrections & $190 \pm 75$ & $126 \pm 71$ & $37 \pm 13$ & $80 \pm 32$ \\
& $-7 \pm 10$ & $-3 \pm 16$ & $-2 \pm 24$ & $-11 \pm 11^{*}$ \\
\hline Sites' HeartModel values with corrections \pm SD & $198 \pm 80$ & $127 \pm 77$ & $40 \pm 15$ & $79 \pm 32$ \\
& & & & \\
Bias (\% mean) \pm SD & $-3 \pm 11$ & $-5 \pm 15$ & $3 \pm 23$ & $-12 \pm 12^{*}$ \\
\hline
\end{tabular}

Table 1. Comparison of the Sites' HeartModel measurements to the Core Lab’s Manual Measurements.

Manual contour corrections of the automatically detected boundaries resulted in minimal changes in the measurements as a percent of the mean measured value (Table 1, row 4) and in the correlations: $r=0.97,0.98$, 0.90, 0.96 for LVEDV, ESV, EF and LAV, respectively. The biases became smaller, with the exception of LAV, and a minimal decrease was noted in the limits of agreement. When expressed as percent of the mean measured value, the biases were $3-7 \%$ for $\mathrm{LV}$ volumes and only $2 \%$ for EF, and were not significant; however, LAV was significantly underestimated by $11 \%$ (Table 1 , rows 3 ). These biases were not clearly affected by contour corrections (Table 1, row 5).

Intra-technique comparisons showed perfect agreement between all automated measurements made by the sites and the CL, when no contour corrections were made. This perfect agreement was reflected by volumes and EF that were identical (Table 2, rows 1 and 2), correlations that were all $\mathrm{r}=1.0$ and biases all zero.

\begin{tabular}{|ccccc|}
\hline & EDV $(\mathrm{ml})$ & $\mathrm{ESV}(\mathrm{ml})$ & $\mathrm{EF}(\%)$ & LAV $(\mathrm{ml})$ \\
\hline $\begin{array}{c}\text { Core Lab's HeartModel values } \\
\text { without corrections }\end{array}$ & $190 \pm 75$ & $126 \pm 71$ & $37 \pm 13$ & $80 \pm 32$ \\
\hline Sites' HeartModel values without corrections & $190 \pm 75$ & $126 \pm 71$ & $37 \pm 13$ & $80 \pm 32$ \\
Bias (\% mean) \pm SD & $0 \pm 0$ & $0 \pm 0$ & $0 \pm 0$ & $0 \pm 0$ \\
\hline $\begin{array}{c}\text { Core Lab's HeartModel values } \\
\text { with corrections }\end{array}$ & $206 \pm 80$ & $133 \pm 78$ & $39 \pm 15$ & $89 \pm 35$ \\
\hline $\begin{array}{c}\text { Sites' HeartModel values with corrections } \\
\text { Bias (\% mean) } \pm \text { SD }\end{array}$ & $198 \pm 80$ & $127 \pm 77$ & $40 \pm 15$ & $79 \pm 32$ \\
\hline
\end{tabular}

Table 2. Comparison of the Sites' HeartModel measurements to the Core Lab’s Manual Measurements.

With contour corrections made by both the sites and the CL, intra-technique comparisons showed excellent agreement with volumes and EF values being similar (Table 2, rows 4 and 5) and the correlations being: $r=0.99,0.99,0.94,0.99$ for LV EDV, ESV, EF and LAV, respectively. These correlations were all higher than in the inter-technique comparisons with contour corrections. In contrast, biases were similar to those noted in the intertechnique comparisons with contour corrections, but importantly the limits of agreement were considerably narrower. The biases were $5-6 \%$ of the measured values for the LV volumes and only 3\% for EF, and $12 \%$ for LAV (Table 2, rows 6).

Overall, readers determined that no contour corrections were needed in 35 (19\%) patients for EDV, 40 (22\%) patients for ESV and in 72 (40\%) patients for LAV, while some contour correction was performed in the remaining patients. Of note, in the majority of the patients only global contour corrections were performed for LV volumes: 98 patients for EDV (54\%) and 90 patients for ESV (50\%), while no global editing is available for LAV. Importantly, the relative changes in volumes caused by contour corrections were $\leq 4 \%$. 
Reproducibility results are presented in Table 3. Importantly, both the inter- and intra-observer variability levels were lower for the automated measurements than the conventional manual technique for all parameters.

\begin{tabular}{|l|c|c|c|c|c|c|c|c|}
\hline \multirow{2}{*}{$\%$ Variability } & \multicolumn{4}{|c|}{$\begin{array}{c}\text { HeartModel } \\
\text { (with corrections when needed) }\end{array}$} & \multicolumn{5}{c|}{ Conventional Manual } \\
\cline { 2 - 9 } & EDV & ESV & EF & LAV & EDV & ESV & EF & LAV \\
\hline Intra-observer & $3 \pm 3$ & $5 \pm 5$ & $7 \pm 9$ & $7 \pm 6$ & $7 \pm 6$ & $8 \pm 9$ & $12 \pm 13$ & $9 \pm 7$ \\
\hline Inter-observer & $6 \pm 5$ & $9 \pm 9$ & $13 \pm 12$ & $11 \pm 6$ & $11 \pm 8$ & $13 \pm 11$ & $17 \pm 14$ & $15 \pm 12$ \\
\hline
\end{tabular}

Table 3. Intra- and inter-observer variability data.

\section{Conclusions}

Although most previously published reports have endorsed earlier automated techniques for 3DE evaluation of LV volumes for clinical use, their conclusions were based on single center studies, in which data were acquired and measured by highly trained personnel [6-7]. To our knowledge, this is the first multicenter study to test the new automated 3DE approach for simultaneous LV and LA volumes and LV function assessment based on an adaptive analytics algorithm. This study shows that experienced readers in different parts of the world can obtain accurate and reproducible automated measurements of LV EDV, ESV and EF, contrary to previous studies that tested other automated algorithms [8]. Moreover, the new automated 3DE algorithm also simultaneously measures LAV without additional time or effort. Unlike 2DE, where dedicated LA focused views are needed to perform accurate bi-plane measurements, with 3DE, the LA is included in the pyramidal dataset and no additional imaging is needed either. Importantly, automated reasonably accurate and reproducible LAV measurements were obtained in this multicenter study.

The inter- and intra-technique comparisons between the automated analyses by the sites against the different reference standards generated by the CL showed that the new methodology can be used universally and provide similar results. An unexpected finding of these analyses was that contour editing, although judged as necessary in the majority of patients, had only limited effects on the measurements. One might suggest that these differences would not be clinically significant, because the largest bias we found in LV EF was 3\% of the measured value, i.e. only $1.2 \%$ (in actual EF units), which is below the accuracy of any technique that measures EF. However, boundary corrections are likely to be important in individual patients, where they can result in considerably larger volume and EF differences that may have important clinical implications.

Another interesting finding was that the automated technique with corrections was more reproducible than the conventional manual technique for all 4 parameters.
This may be related to the fact that the automated software starts from endocardial border position that is based on algorithm settings that were optimized on thousands of patients. In addition, the automated software identifies the ED and ES frames, unlike the manual analysis, wherein the user needs to select the correct frames. In this study, the readers were instructed to use for their manual analyses the frames chosen by the automated software, in order to eliminate this source of variability. Therefore, our study underestimated the true variability of the conventional methodology, which further underscores the value of the automated approach.

In summary, automated volumetric analysis of leftheart chambers is an accurate and robust alternative to conventional manual methodology, which yields almost the same values across laboratories and is more reproducible. This technique may contribute towards full integration of 3DE quantification into clinical routine.

\section{References}

[1] Mor-Avi V, Jenkins C, Kuhl HP, et al. Real-time 3dimensional echocardiographic quantification of left ventricular volumes: Multicenter study for validation with magnetic resonance imaging and investigation of sources of error. JACC Cardiovasc Imaging. 2008;1:413-23.

[2] Mor-Avi V, Yodwut C, Jenkins C, et al. Real-time 3D echocardiographic quantification of left atrial volume: Multicenter study for validation with CMR. JACC Cardiovasc Imaging. 2012;5:769-77.

[3] Lang RM, Badano LP, Mor-Avi V, et al. Recommendations for cardiac chamber quantification by echocardiography in adults: an update from the ASE and EACVI. J Am Soc Echocardiogr. 2015;28:1-39.

[4] Muraru D, Badano LP, Ermacora D, et al. Sources of variation and bias in assessing left ventricular volumes and dyssynchrony using 3D echocardiography. Int J Cardiovasc Imaging. 2012;28:1357-68.

[5] Tsang W, Salgo IS, Medvedofsky D, et al. Transthoracic three-dimensional echocardiographic left heart chamber quantification using an automated adaptive analytics algorithm. JACC Cardiovasc Imaging. 2016 (in press).

[6] Chang SA, Lee SC, Kim EY, et al. Feasibility of singlebeat full-volume real-time 3D echocardiography and autocontouring algorithm for quantification of left ventricular volume. J Am Soc Echocardiogr. 2011;24:853-9.

[7] Jenkins C, Leano R, Chan J et al. Reconstructed versus real-time 3D echocardiography: comparison with MRI. J Am Soc Echocardiogr. 2007;20:862-8.

[8] Shimada YJ, Shiota T. A meta-analysis and investigation for the source of bias of left ventricular volumes and function by 3D echocardiography in comparison with MRI. Am J Cardiol. 2011;107:126-38.

Address for correspondence:

Victor Mor-Avi, PhD

University of Chicago Medical Center, M.C. 9067

5758 S Maryland Ave., 60637 Chicago, IL, USA

vmoravi@bsd.uchicago.edu 